\title{
Insémination artificielle intra-utérine sous contrôle laparoscopique chez les petits ruminants domestiques
}

\author{
JC Vallet 1, 3, G Baril 1, B Lebœuf 2, J Perrin 3 \\ ${ }^{1}$ INRA, physiologie de la reproduction et URA CNRS 1291, 37380 Nouzilly; \\ 2 INRA-SEIA, 86480 Rouillé; \\ 3 Procreatech SA, ZI de la Centrale, 81400 Carmaux, France
}

(Reçu le 4 juin 1991; accepté le 2 septembre 1991)

\begin{abstract}
Résumé - Depuis quelques années, la technique d'IA intra-utérine sous contrôle laparoscopique a été décrite par différents auteurs chez les ovins et les caprins de race Angora et Cachemire. Pour ces espèces, l'intérêt de cette technique est d'augmenter le pouvoir de diffusion des mâles améliorateurs par l'utilisation de faibles quantités de semence congelée, déposée in utero, tout en assurant des niveaux de fertilité satisfaisants. Plus récemment, cette méthode a été testée chez les caprins laitiers. Au cours des 2 dernières campagnes d'IA, 12000 ovins ont été inséminés en France par cette méthode. Notre propre expérience a porté sur la mise en place de 1112 doses de semence ovine congelée de race Rouge de l'Ouest et Vendéenne (1989 et 1990) et, à titre expérimental, sur 334 inséminations (1990) de caprins laitiers (Alpins et Saanen). Les résultats obtenus sont respectivement de $70,7 \%$ de fertilité et 1,88 agneaux par mise bas chez les ovins, et $53,5 \%$ de fertilité, comparés à $42,9 \%$ pour la méthode classique d'IA dans les mêmes troupeaux, chez les caprins.
\end{abstract}

insémination artificielle / utérus / brebis / chèvre / fertilité

Summary - Intra-uterine insemination by laparoscopy in ewes and goats. The technique of intra-uterine artificial insemination (Al) under laparoscopic control has been described recently by different authors in ovine species, that is, in Angora and Cashmere goats. For both species the interest of this technique is to increase the diffusion rate of genetically selected sires by using low quantities of deep-frozen semen deposited in utero and obtaining satisfactory levels of fertility. More recently, this technique has been tested in dairy goats. During the last 2 Al campaigns, 12000 ewes were inseminated in France by this method. Our own experiment consisted of Al using 1112 doses of ovine deep-frozen semen of Rouge de l'Ouest and Vendéenne breeds (1989 and 1990) and at an experimental level, on 334 inseminations (1990) of dairy goats (Alpine and Saanen). In the goat, results obtained were $70.7 \%$ fertility and 1.88 lambs per lambing in ewes, and $53.5 \%$ fertility, compared to $42.9 \%$ for the classical Al method applied in the same flocks.

artificial insemination / uterus / ewe/ goat / fertility 


\section{INTRODUCTION}

Depuis de nombreuses années, l'insémination artificielle a été, chez les ruminants domestiques l'outil indispensable du progrès génétique, c'est ainsi que 650 à 700000 petits ruminants sont inséminés chaque année en France depuis 1988, à partir de doses de semence fraîche ou congelée.

La mise au point de nouvelles techniques de reproduction et, en particulier, l'approche de l'utérus par la voie transpéritonéale sous contrôle endoscopique permettent l'obtention de taux de fertilité satisfaisants, après mise en place de nombres réduits de spermatozoïdes congelés (Maxwell et al, 1984). En 1990, 6000 brebis furent inséminées en France, principalement dans les élevages intégrés à un schéma de sélection. Chez les caprins, des expéri-

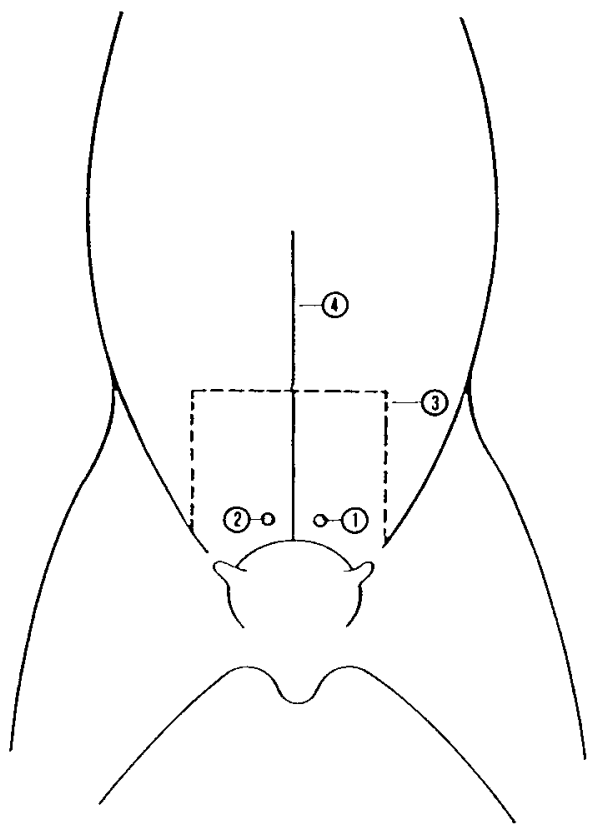

Fig 1. Sites d'insertion des trocards de $7 \mathrm{~mm}$ (1) et de $5 \mathrm{~mm}$ (2), par rapport au champ opératoire (3) et à la cligne blanche" médiane (4). mentations sont en cours depuis 2 ans afin de préciser l'efficacité de la méthode dans cette espèce.

\section{MATÉRIEL ET MÉTHODES}

\section{Traitements de synchronisation de l'œstrus}

Les ovulations sont induites après traitement de synchronisation de l'œstrus (éponges vaginales : $30-40-45 \mathrm{mg}$ d'acétate de fluorogestone) par une injection de PMSG (pregnant mare serum gonadotrophin). Ces traitements varient en fonction de l'espèce, de la race, de la saison, de la production laitière et de la parité des femelles. Chez la chèvre, une prostaglandine (PGF2 $\alpha$ ) est injectée $48 \mathrm{~h}$ avant le retrait du support progestatif simultanément à l'injection de PMSG.

\section{Technique d'insémination sous contrôle endoscopique}

L'endoscopie est une technique d'exploration interne incluant un système optique utilisant les propriétés de propagation de la lumière dans une fibre de verre. L'endoscopie, terme général désignant cette technologie est appelée laparoscopie, lorsqu'elle s'adresse à l'exploration de la cavité abdominale préalablement distendue par un pneumopéritoine artificiel (insufflation d'air ou de $\mathrm{CO}_{2}$ ). Préalablement aux inséminations, les femelles sont soumises à une diète de $24 \mathrm{~h}$ afin de réduire l'encombrement du tractus digestif, et de faciliter la localisation et la manipulation instrumentale de l'appareil génital. Les inséminations sont réalisées en plaçant l'animal en décubitus dorsal, crânialement incliné à $45^{\circ}$, le champ opératoire est tondu puis désinfecté, les zones de ponction sont insensibilisées par une anesthésie locale. L'introduction des trocards s'effectue de part et d'autre de la ligne blanche (fig 1). Après mise en place des instruments et la localisation du tractus génital (fig 2), la semence est déposée dans chacune des cornes utérines. Les trocards sont ensuite retirés, un antibiotique est appliqué sur chaque point de ponction. 


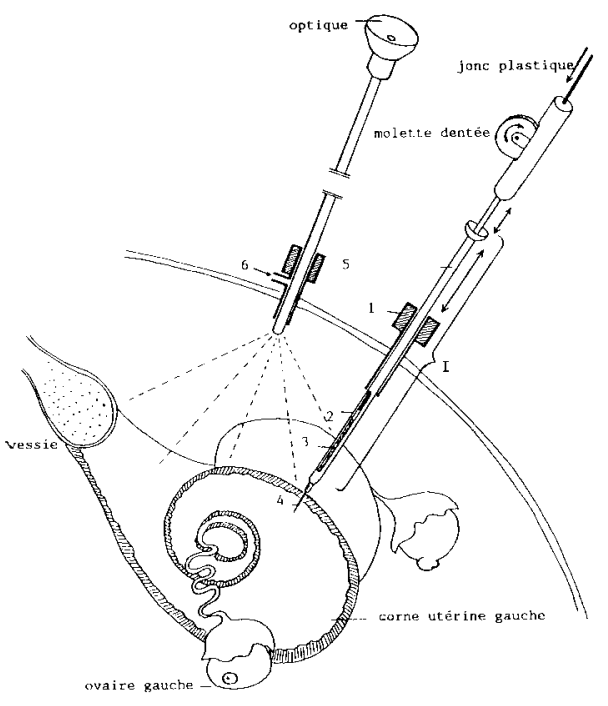

Fig 2. Descriptif de la technique d'insémination intra-utérine sous contrôle laparoscopique ( $1=$ palpateur; 1 = canule de diam $5 \mathrm{~mm}$; 2 = lumière du palpateur; 3 = paillette fine $(0,25 \mathrm{ml}) ; 4$ = aiguille long $5 \mathrm{~mm}$ diam $0,7 \mathrm{~mm} ; 5$ = canule de diam $7 \mathrm{~mm}$; 6 = gaz du pneumopéritoine).

\section{Chez les caprins}

Au cours des campagnes 1989-1990, 2 expérimentations ont été menées d'abord pour essayer d'améliorer la fertilité par l'utilisation de I'IA sous contrôle laparoscopique en utilisant de très faibles nombres de spermatozoïdes, ensuite pour tester l'effet de dilution avant congélation à $-196{ }^{\circ} \mathrm{C}$ sur la fertilité après dégel des spermatozoïdes de bouc. Ces 2 expérimentations ont été réalisées dans la proportion 1/3 IA cervicale "classique" vs $2 / 3$ IA intra-utérine dans les mêmes troupeaux $(n=7)$. Les inséminations cervicales et sous contrôle laparoscopique ont eu lieu $43 \pm 1 \mathrm{~h}$ (Alpins) et $45 \pm 1 \mathrm{~h}$ (Saanen) après le retrait de l'éponge.

En 1989, les femelles inséminées par voie cervicale $(n=75)$ l'ont été avec $200 \times 10^{6}$ sper- matozoïdes totaux (spzt), celles inséminées par laparoscopie avec $10(n=91)$ ou $20 \times 10^{6}$ $(n=92)$ spzt. Cette comparaison n'a pas été effectuée à partir d'éjaculats partagés.

En 1990, les femelles inséminées par voie cervicale $(n=166)$ l'ont été avec $100 \times 10^{6} \mathrm{spzt}$ (race Alpine: AL) ou $60 \times 10^{6}$ spzt (Saanen : AA). Les inséminations intra-utérines $(n=334)$ ont été réalisées pour la moitié de l'effectif $(n=163)$ à partir de paillettes fines contenant initialement $20 \times 10^{6} \mathrm{spzt}$ (AL-AA) et pour l'autre moitié $(n=171)$ à partir de paillettes fines contenant $100(\mathrm{AL})$ ou $60 \times 10^{6} \mathrm{spzt}$ (AA) fractionnées après redilution pour obtenir des doses de $20 \times 10^{6}$ spzt. Pour l'année 1990, 198 inséminations ont été réalisées en dehors de l'expérimentation dont 62 IA cervicales et 136 IA sous contrôle laparoscopique (effectuées dans les mêmes troupeaux).

\section{Chez les Ovins}

Les inséminations ont été réalisées en routine au cours des années 1989-1990. Elles ont été effectuées dans les races vendéenne et Rouge de l'Ouest avec des doses de semences congelées de $100 \times 10^{6} \mathrm{spzt}$ congelés totaux. Les inséminations ont eu lieu $55 \mathrm{~h} \pm 1 \mathrm{~h}$ après le retrait de l'éponge.

Dans les 2 espèces, les résultats de fertilité sont exprimés en pourcentage de femelles mettant bas.

\section{RÉSULTATS}

\section{Caprins}

En 1989, la fertilité après IA sous contrôle laparoscopique est significativement supérieure à celle observée après IA cervicale $(62,6$ vs $49,3 \% ; P<0,05)$. La fertilité après IA sous contrôle laparoscopique ne diffère pas significativement selon le nombre de spermatozoïdes mis en place $\left(10 \times 10^{6}\right.$ spzt : $61,5 \%$ vs $20 \times 10^{6}$ spzt : $63,7 \%$; tableau I). 
Tableau I. Fertilité, en pourcentage de mise bas et prolificité après IA cervicale ou intra-utérine par endoscopie chez la chèvre laitière (campagne 1989). a est différent de b au seuil de $P<$ 0,05 .

\begin{tabular}{llll}
\hline & IA cervicale & \multicolumn{2}{c}{ IA intra-utérine } \\
\hline $\begin{array}{llll}\text { Nombre } \\
\text { de spz }\end{array}$ & $200 \times 10^{6}$ & $10 \times 10^{6}$ & $20 \times 10^{6}$ \\
Fertilité \% & $49,3^{\mathrm{a}}$ & 61,5 & 63,7 \\
(nombre d'IA) & $(75)$ & $(91)$ & $(91)$ \\
& \multicolumn{4}{c}{$\begin{array}{c}\text { Total } \\
62,6^{\mathrm{b}}\end{array}$} \\
& \multicolumn{4}{c}{$(182)$} \\
Prolificité & 2,03 & 2,06 & 2,02 \\
\hline
\end{tabular}

En 1990, pour l'ensemble des 2 races, la fertilité après IA intra-utérine est significativement plus élevée que celle obtenue après IA cervicale $(44,3$ vs $34,3 \%$; $P<0,05)$. Quelle que soit la race, la fertilité après IA intra-utérine par laparoscopie avec $20 \times 10^{6}$ spzt n'est pas significativement différente selon la concentration initiale en spermatozoïde à la congélation (tableau II).

Pour l'ensemble des inséminations effectuées en 1989-1990, le pourcentage de mises-bas observé après IA laparoscopie est très significativement supérieur (environ $10 \%$ ) à celui obtenu après IA cervicale ( 53,5 vs $42,9 \%$ de fertilité, pour 652 et 303 IA, respectivement).

\section{Ovins}

Dans les races Rouge de l'Ouest et Vendéenne, la fertilité après IA intra-utérine avec de la semence congelée est respectivement de $71,3 \%$ et $68,2 \%$ et la prolificité de $189,3 \%$ et $184,2 \%$ (tableau III).

Tableau II. Fertilité, en pourcentage de mise bas après IA cervicale ou intra-utérine par endoscopie chez la chèvre laitière, selon la race et le nombre de spermatozoïdes mis en place (campagne 1990). Le résultat noté a est différent du résultat noté b au seuil de $P<0,05$.

IA cenvicale

Dose de semence $\left(\times 10^{6} \mathrm{spz}\right) \quad 60$

100

20

$20^{1}$

$20^{2}$

Race

alpine

(nombre d'IA)

saanen

(nombre d'IA)

29,5

(88)

39,7

(78)

49,3

(75)

51,3

(78)

Total 2 races

${ }^{1}$ paillette de $60 \times 10^{6} \mathrm{spz}$ fractionnées en $20 \times 10^{6} \mathrm{spz} ;{ }^{2}$ paillettes de $100 \times 10^{6} \mathrm{spz}$ fractionnées en $20 \times 10^{6} \mathrm{spz}$ 
Tableau III. Fertilité, en pourcentage de mise bas après insémination intra-utérine de $100 \times$ $10^{6} \mathrm{spz}$ totaux par endoscopie chez la brebis.

\begin{tabular}{|c|c|c|c|}
\hline \multirow[t]{2}{*}{ Race } & \multicolumn{2}{|c|}{ Fertilité } & \multirow{2}{*}{$\begin{array}{l}\text { Prolificité } \\
\text { agnx/ } \\
\text { brebis }\end{array}$} \\
\hline & $(\%)$ & $(N b / A)$ & \\
\hline Rouge de l'ouest & 71,3 & 898 & 1,89 \\
\hline Vendéenne & 68,2 & 214 & 1,84 \\
\hline Total deux races & 70,7 & 1112 & 1,88 \\
\hline
\end{tabular}

\section{CONCLUSION}

Chez les caprins, l'utilisation de la technique d'IA sous contrôle laparoscopique permet d'augmenter le pourcentage de mise- bas de $10 \%$ et de réduire le nombre de spermatozoïdes mis en place. Chez les ovins, seule I'IA par laparoscopie permet d'obtenir un niveau satisfaisant de fertilité après mise en place de spermatozoïdes congelés. Pour les 2 espèces, cette technique conduit à une augmentation importante du pouvoir de diffusion des meilleurs mâles et doit permettre de retenir, dans les schémas de testage, des mâles de niveau génétique élevé mais dont la production quantitative de spermatozoïdes était jusqu'alors considérée comme insuffisante pour une diffusion par insémination traditionnelle.

\section{RÉFÉRENCE}

Maxwell WMC, Butler LG, Wilson HR (1984) Intra-uterine insemination of ewes with frozen semen. J Agric Sci (Camb) 102, 233-235 